\section{Quand le développement du cerveau façonne l'individualité comportementale}

> Qu'est-ce qui fait de nous des individus? L'individualité fait référence aux caractères qui permettent de différencier un individu d'un autre au sein d'une même espèce. Tenter de comprendre l'origine de l'individualité est un objectif central partagé par de nombreuses disciplines allant de la psychologie à la neurobiologie, la génétique ou encore l'écologie. Outre les différences portant sur des caractères anatomiques, il existe également des différences de comportement qui persistent au cours du temps, et qui concernent les vertébrés comme les invertébrés [1]: on parle d'individualité comportementale.

L'individualité comportementale chez la drosophile

Pour mieux comprendre les mécanismes à l'origine de l'individualité comportementale, nous nous sommes intéressés à un comportement inné dans une espèce invertébrée: la capacité de la mouche drosophile (Drosophila melanogaster) à fixer un objet au cours de son déplacement. Conservée chez les animaux et chez l'homme, cette capacité fait appel aux propriétés générales du système visuel. Elle peut être mesurée grâce au test de l'arène de Buridan. Simple et reproductible, il permet de tester un même individu plusieurs fois au cours du temps (Figure 1). Le dispositif est composé d'une arène circulaire entourée de parois blanches sur lesquelles deux bandes noires verticales se font face. Une mouche placée au centre peut marcher librement dans l'espace et choisir la direction de ses déplacements.
Attirée par les bandes noires, elle ne pourra néanmoins pas les atteindre et entamera des allers-retours entre les deux objets. Sur une population de drosophiles testées une à une, certaines suivent une trajectoire précise entre les deux stimulus, caractérisée par un faible angle de déviation par rapport à l'axe défini par ces stimulus. D'autres, en revanche, adoptent un comportement exploratoire, avec un angle de déviation plus ou moins grand selon les individus testés. Ainsi, au sein d'une population de mouches, chaque individu a une trajectoire de déplacement qui lui est propre, et ce comportement sera constant tout au long de sa vie [2].

\section{Une individualité non-héréditaire}

Comment expliquer cette individualité comportementale? Qu'ils soient génétiques, environnementaux ou autres, différents facteurs peuvent être à l'origine d'une individualité comportementale. Dans le cas d'une contribution génétique, chaque caractère individuel sera le reflet de variations génétiques au sein de la population. La sélection d'un phénotype spécifique au cours des générations devrait entraîner une homogénéisation de la population pour ce caractère. Cependant, le croisement successif d'individus présentant le même phénotype comportemental, par exemple un déplacement avec un faible angle de déviation, génère une diversité phénotypique dans la descendance: l'ensemble des angles de déplacement est recréé au sein de la population. Le caractère spécifique des
${ }^{1}$ Institut du cerveau et de la moelle épinière (ICM) - Sorbonne Université, Inserm, CNRS, Hôpital Pitié-Salpêtrière, 47 boulevard de l'Hôpital, 75013 Paris, France.

${ }^{2}$ Division of neurobiology of the Institute for biology, Free university, Königin-Luise-Str.

1-3, DE 14195 Berlin, Allemagne.

${ }^{3}$ Einstein-BIH, Charité Universitätsmedizin, Berlin, Allemagne.

maheva.andriatsilavo@charite.de bassem.hassan@icm-institute.org

parents n'est donc pas transmis aux générations suivantes. De même, une réduction de la diversité génétique de la population n'entraîne pas de diminution de la diversité phénotypique. Le caractère individuel de la drosophile à fixer un objet au cours de son déplacement n'est donc pas lié à un facteur génétique [2]. Un phénomène similaire est observé lorsque des drosophiles sont confrontées au choix de tourner à gauche ou à droite dans un labyrinthe à deux entrées [3]. Certains individus iront de préférence à gauche, d'autres à droite. D'autres encore n'auront pas de préférence et choisiront aléatoirement l'une des deux directions. Ces préférences individuelles sont constantes dans le temps et ne sont pas héréditaires.

\section{Une corrélation entre le degré \\ d'asymétrie du cerveau et \\ l'individualité comportementale}

Des enchevêtrements de neurones cérébraux interconnectés en réseaux sont à l'origine de nos comportements. Pour comprendre pourquoi chaque drosophile possède une capacité à fixer un objet qui lui est propre, nous nous sommes intéressés aux circuits neuronaux impliqués dans ce comportement: les neurones du cluster dorsal (dorsal cluster neurons, DCN) (Figure 1). Les axones de ces neurones commissuraux traversent la commissure centrale du cerveau de la mouche pour atteindre le lobe optique opposé [4] et innerver une première zone proximale, la lobula, tandis qu'une partie de ces axones va également innerver une seconde structure 


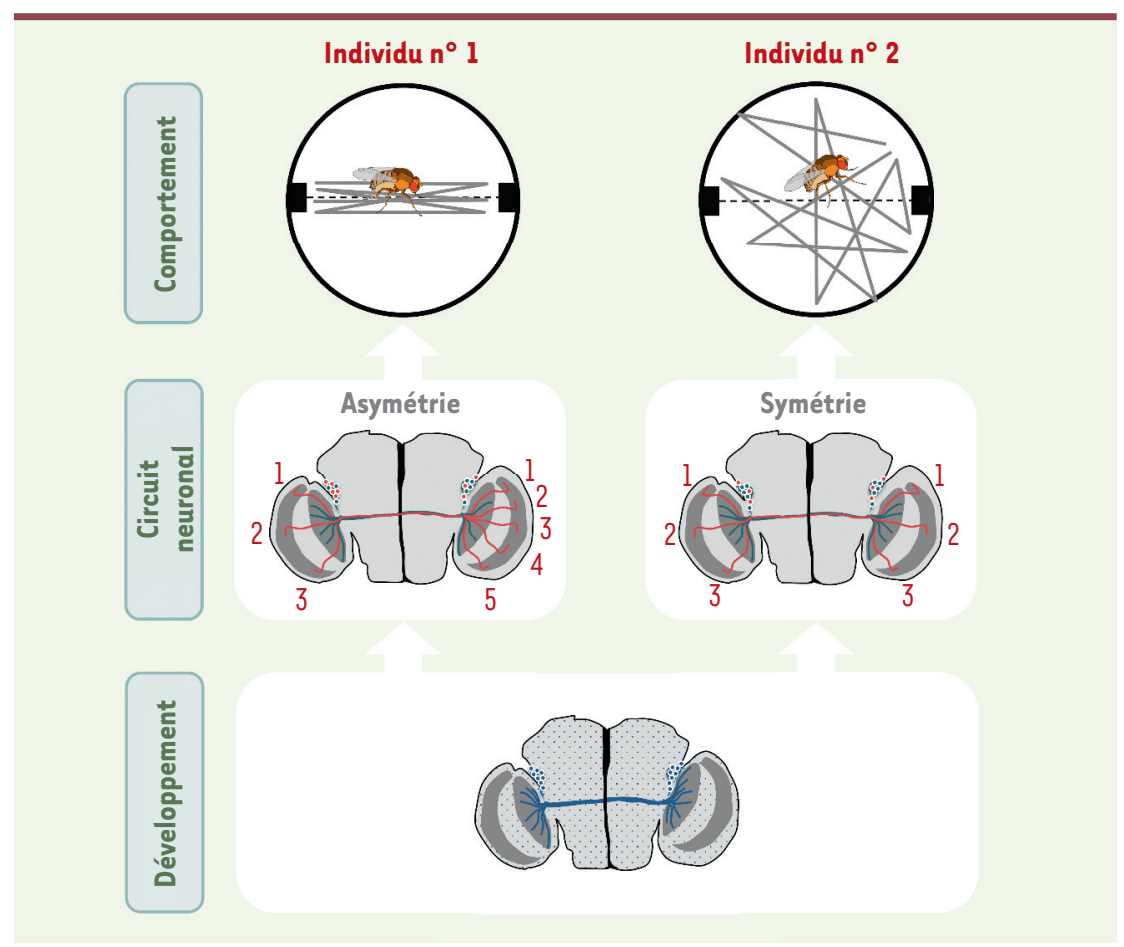

Figure 1. Une origine développementale de l'individualité comportementale chez la mouche drosophile. Le circuit des neurones du cluster dorsal ( $D C N$, ici représenté en vert et en rouge) est impliqué dans la capacité des drosophiles à fixer un objet au cours d'un déplacement dans l'arène de Buridan. Ce comportement inné varie d'un individu à l'autre pour un même environnement et un même fonds génétique. Les axones des neurones DCN traversent la commissure centrale du cerveau pour atteindre le lobe optique opposé, où l'ensemble des axones (représentés en rouge et en vert) innervent une zone proximale : la lobula. Une partie de ces axones (représentés en vert) va également innerver une structure distale, la medulla. Au cours du développement de ce circuit neuronal (représenté en bleu dans le schéma du bas), la zone d'innervation d'un axone est déterminée de manière aléatoire par l'intermédiaire de l'activation ou non de la voie de signalisation Notch, avec pour conséquence de générer un circuit neuronal présentant une asymétrie structurelle droite-gauche propre à chaque individu. Cette différence anatomique entre individus est à l'origine d'une individualité comportementale dans la capacité de fixation des objets.

distale, la medulla, qui est impliquée dans la détection des mouvements et des couleurs. Le nombre total de neurones DCN, le nombre d'axones innervant la medulla, et le degré de ramification de chaque axone varient d'un individu à l'autre, et entre les deux lobes optiques d'un même animal $[5,6]$. Un degré d'asymétrie peut alors être défini pour chaque individu, en prenant en compte l'ensemble des paramètres morphologiques des circuits gauches et droits des neurones dorsaux. Plus le degré d'asymétrie est élevé, plus l'individu établit une trajectoire précise entre les deux (asymmetric body), qui est impliqué dans la formation de la mémoire à long terme et n'est présent que dans un seul des hémisphères cérébraux chez la grande majorité des individus [7]. Cependant, chez une faible proportion d'individus, le corpus asymétrique est présent dans les deux hémisphères, et ces mouches au cerveau symétrique ont une moins bonne mémoire que leurs congénères. L'asymétrie du cerveau et son impact sur les capacités cognitives d'un individu ne sont d'ailleurs pas restreints à la drosophile [1]. Ainsi, comprendre l'origine de l'individualité comportementale nécessite de comprendre comment se constitue cette asymétrie dans les circuits neuronaux.

\section{Une origine développementale de l'individualité comportementale} Dans le contexte du débat sur l'origine de nos différences, «l'inné », souvent associé à une origine génétique, est mis en opposition avec «l'acquis », qui définit les traits influencés par l'expérience. Tout ce qui n'est pas défini génétiquement est souvent associé à une origine environnementale. Cependant, lorsque des individus avec le même patrimoine génétique sont soumis à un même environnement, des différences anatomiques et comportementales persistent, et il en est de même lorsque I'on compare les côtés gauche et droit du cerveau chez un même animal. Chez l'homme, les empreintes digitales de chaque doigt sont différentes, l'innervation de la main et la forme de la rétine varient. Une troisième source à l'origine de nos différences est souvent oubliée: ce sont les processus cellulaires et moléculaires mis

en place au cours

$(\rightarrow)$ Voir le Forum de V. Debat, $m / s n^{\circ} 8-9$, du développement août-septembre 2016, $[8,9](\rightarrow)$. page 774 existe ainsi une corrélation entre l'individualité comportementale et l'asymétrie du circuit des neurones DCN [2]. L'asymétrie concerne également une structure particulière du cerveau de la drosophile, le corpus asymétrique
Cela s'applique également pour le développement du cerveau, qui présente une asymétrie anatomique de ses deux hémisphères [5-7]. Grâce à l'utilisation d'une technique d'imagerie intravitale, nous avons pu montrer que les circuits 
neuronaux des DCN du cerveau de la drosophile, et donc les variations structurelles individuelles, sont établis au cours du développement, et persistent au stade adulte [2].

Au cours du développement, une perturbation des signaux impliqués dans la formation du circuit des neurones DCN affecte la mise en place du réseau neuronal final. Par exemple, une inhibition de l'endocytose pendant la période critique d'innervation de la medulla augmente la proportion de circuits symétriques au sein de la population de mouches, et la proportion d'individus qui auront un déplacement approximatif dans l'arène de Buridan [2]. Le développement des circuits des neurones DCN conditionne donc le comportement des futurs adultes.

\section{Une détermination aléatoire de l'asymétrie neuronale}

Comment ces variations structurelles à l'origine d'une individualité comportementale sont-elles établies au cours du développement? De nombreuses voies de signalisation interagissent dans la formation du circuit des neurones DCN [4-6]. En amont de ces signaux, l'activation ou non de la voie du récepteur Notch détermine la zone d'innervation finale d'un axone. Une activation de cette voie dans un neurone DCN immature entraîne une innervation de la lodula. À l'inverse, une inhibition de la voie Notch augmente la probabilité d'un axone d'innerver la medulla [5]. Cette activation se fait par le biais d'une interaction entre le récepteur Notch et son ligand transmembranaire Delta. Delta permet d'envoyer un signal activateur de la voie Notch aux cellules voisines. Cette activation de la voie Notch dans les cellules receveuses réduit la production de Delta et donc leur capacité d'émettre un signal d'activation. Les cellules émettrices, quant à elles, n'activeront pas la voie Notch. Au cours du développement des neurones DCN, la voie Notch est au cœur d'un mécanisme d'inhibition mutuelle entre cellules [10]. À la fois émetteur et receveur, chaque neurone DCN immature entre en compétition avec ses neurones voisins pour l'activation de la voie Notch. Les différences de niveau d'activation de la voie Notch entre neurones DCN sont alors amplifiées. À l'issue de cette interaction cellulaire, seule une partie des neurones DCN activera la voie Notch et innervera la lobula. Les autres n'activeront pas la voie Notch et innerveront la medulla [5]. La zone d'innervation de l'axone d'un neurone DCN n'est donc pas prévisible. Elle est déterminée aléatoirement par le biais de l'interaction entre le récepteur Notch et son ligand Delta [5]. La mise en place de ce circuit neuronal n'est pas le fruit d'une simple addition entre l'expression des gènes et l'environnement: elle résulte également du caractère aléatoire des mécanismes cellulaires et moléculaires mis en place au cours du développement. Ces résultats obtenus chez la mouche drosophile montrent l'importance de ces processus développementaux dans le fonctionnement du cerveau et dans l'émergence d'une individualité cognitive et comportementale. $\diamond$

When brain development shapes individual behavior

\section{LIENS D'INTÉRÊT}

Les auteurs déclarent n'avoir aucun lien d'intérêt concernant les données publiées dans cet article.

\section{RÉFÉRENCES}

1. Forrester GS, Hopkins WD, Hudry K, et al. Cerebral lateralization and cognition: evolutionary and developmental investigations of behavioral biases. Prog Brain Res $2018 ; 238: 457$.

2. Linneweber GA, Andriatsilavo M, Dutta SB, et al. A neurodevelopmental origin of behavioral individuality in the Drosophila visual system. Science 2020 ; 367 : 1112-9.

3. Buchanan SM, Kain JS, de Bivort BL. Neuronal control of locomotor handedness in Drosophila. Proc Natl Acad Sci USA 2015 ; 112 : 6700-5.

4. Srahna M, Leyssen M, Ching MC, et al. A signaling network for patterning of neuronal connectivity in the Drosophila brain. PLoS Biol 2006 ; 11 : e348.

5. Langen M, Koch M, Yan J, et al. Mutual inhibition among postmitotic neurons regulates robustness of brain wiring in Drosophila. Elife $2013 ; 2$ : e00337.

6. Zschätzsch M, Oliva C, Langen M, et al. Regulation of branching dynamics by axon-intrinsic asymmetries in tyrosine kinase receptor signaling. Elife 2014 ; 3 : e01699.

7. Pascual A, Huang K-L, Neveu J, et al. Brain asymmetry and long-term memory. Nature 2004 ; 427 : 605-6.

8. Honegger K, de Bivort B. Stochasticity, individuality and behavior. Curr Biol 2018 ; 28 : R8-R12.

9. Debat V. Symmetry is beauty - Or is it? Grandeur et décadence de l'asymétrie fluctuante. Med Sci (Paris) 2016 ; $32: 774-80$.

10. Sjöqvist M, Andersson ER. Do as I say, Not(ch) as I do: lateral control of cell fate. Dev Biol 2019; 447 $58-70$.

\section{AMPS (Association Médecine Pharmacie Sciences)}

La période de candidature pour la passerelle permettant d'intégrer directement les études de Santé (Médecine, Pharmacie, Odontologie et Maïeutique) après un diplôme de niveau Bac +5 ou plus approche à grand pas. Pour cette occasion, l'AMPS (Association Médecine Pharmacie Sciences) a le plaisir de vous présenter son guide de candidature au concours passerelle, ainsi que son guide pour préparer sa rentrée directement en $2^{\mathrm{e}}$ ou $3^{\mathrm{e}}$ année des études de Santé, rédigé par des étudiants qui s'y trouvent déjà

Si vous êtes un candidat potentiel, ou que vous en connaissez, n’hésitez pas à les utiliser, ils contiennent théoriquement toutes les informations utiles pour passer du la candidature à la rentrée dans l'année supérieure. Et n’hésitez pas non plus à nous faire part de toutes vos remarques concernant ces guides, nous nous ferons un plaisir de les améliorer.

Enfin, si vous-même avez bénéficié de cette passerelle par le passé, n’hésitez pas à nous contacter pour nous faire part de vos témoignages, vos successeurs vous en remercieront grandement.

Vous trouverez toutes les informations intéressantes sur le site de l'AMPS : https://amps-asso.org/ > Opportunités > Guides Yanis Bendjelal, rédacteur en chef des guides Sciences-Santé de l'AMPS 